\title{
IMPLEMENTATION OF AGRICULTURAL AUTOMATION SYSTEM USING WEB \& GSM TECHNOLOGIES
}

\author{
Vidadala Srija ${ }^{1}$, P.Bala Murali Krishna ${ }^{2}$ \\ ${ }^{1} P G$ student, Dept of ECE, Sri Mittapalli Institute of Technology for Women, A.P, India, \\ vidadalasrija@gmail.com \\ ${ }^{2}$ Professor, Dept of ECE, Sri Mittapalli Institute of Technology for Women, A.P, India, \\ pbmk05@gmail.com
}

\begin{abstract}
This project probes into the implementation of agricultural automation system using WEB and GSM technologies. This Embedded project is to design and develop a low cost system which is based on embedded platform for agricultural automation. Optimum usage of water is main objective of this system. This project uses soil moisture sensor and temperature sensor to detect the water quantity present in agriculture and water level sensor is used for detecting water level in tank. In this system we monitor status of the sensors through WEB and GSM technologies. Here temperature, soil moisture and water level can be monitored on web page through micro controller and information will be send by SMS. This page contains all the information about the status of the sensors. This information will be viewed at remote location by using GPRS technology.
\end{abstract}

Key words: WEB and GSM technologies, Embedded platform, Soil moisture sensor, Temperature sensor. $* * *$

\section{INTRODUCTION}

India is an agricultural country. The Indian economy is basically agrarian. In spite of economic development and industrialization, agriculture is the backbone of the Indian economy Agriculture uses most of available fresh water resources and this use of fresh water resources will continue to be increases Because of population growth and increased food demand. Increased labour costs, stricter Environmental regulations and increased competition for water resources from urban areas Provide strong motivation for efficient Irrigation system.

This paper presents implementation of agricultural automation system using WEB and GSM technologies. This project was developed to optimize water use for agricultural crops. Here we are using different sensors which are placed at root zones of the field. By using the ZIGBEE technology status of the sensors will be transferred to field information unit. We get all the results by using GSM and GPRS technologies. GSM is used for sending the message and GPRS is used for displaying the values in the webpage.

The rest of the paper is organized as follows. The below section2 describes about some previous irrigation systems. Section 3 describes about overview of proposed system and various blocks present in proposed system.section4 describes about working and results of proposed system. Section5 concludes the paper with an idea to implement the same as a real time project.

\section{AN OVERVIEW OF SOME PREVIOUS IRRIGATION SYSTEM}

The automated irrigation system [1] developed proves that the use of water can be diminished for a given amount of fresh biomass production. The use of solar power in this irrigation system is pertinent and significantly important for organic crops and other agricultural products that are geographically isolated. The Internet link allows the supervision through mobile telecommunication devices, such as a Smartphone.

The automatic irrigation and fertigation system [2] that has been implemented at relatively low cost. In this project ZIGBEE network is used. The simulation is done with the help of Keil $\mathrm{C} \mu$ vision software. In this system ZIGBEE protocols replaces the wired irrigation system. This system proves to be a real time feedback control system which monitors and controls all the activities of irrigation system efficiently.

Embedded System Design for Irrigating Field with Different Crops Using Soil Moisture Sensor [3] be used to implement efficient irrigation scheme for the field having different crops. The system can be further enhanced by using fuzzy logic controller. The fuzzy logic scheme is used to increase the accuracy of the measured value and assists in decision making.

Programmable Irrigation Control System Using Li-Fi [4] developed proves that it has low cost. In addition other applications such as temperature monitoring in compost production can be easily implemented. The LI-FI communication and Internet connection allows the data inspection in real time on a website. 
Irrigation System Using a Wireless Sensor Network and GPRS [5] can be adjusted to minimum maintenance. Using this system we can monitor the status of all the sensors (Soil-moisture, Temperature, Water level) and also the ON/OFF status of the motor and Fan

The some system highlights the development of temperature and soil moisture sensor that can be placed on suitable locations on field for monitoring of temperature and moisture of soil, the two parameters to which the crops are susceptible. The sensing system is based on a feedback control mechanism with a centralized control unit which regulates the flow of water on to the field in the real time based on the instantaneous temperature and moisture values [6].

Automation of Irrigation System Using ANN based Controller [7] described a simple approach to Irrigation control using Artificial Neural Network Controller. It is noteworthy that ANN based systems can save lot of resources (energy and water) and can provide optimized results to all type of agriculture areas.

\section{AN OVERVIEW OF PROPOSED SYSTEM}

The proposed system has two main units one is field sensor unit and another is field information unit. Field sensor unit transmits the sensors data to the field information unit. Here field information unit receives sensor data from field sensor unit.

\subsection{Field Sensor Unit}

This section describes about field sensor unit. The below Fig 1. Shows the block diagram of a field sensor unit. A field sensor unit contains, LPC2148 micro-controller, ZIGBEE module, different sensors, LCD display, motor and power supply circuit. Several field sensor units are placed in-field to configure sensor network for the automated irrigation system. The design of this proposed system is explained as follows. Sensor units are placed at root zone of the field. The below Fig 2. Shows field sensor unit to monitor status of all the sensors.

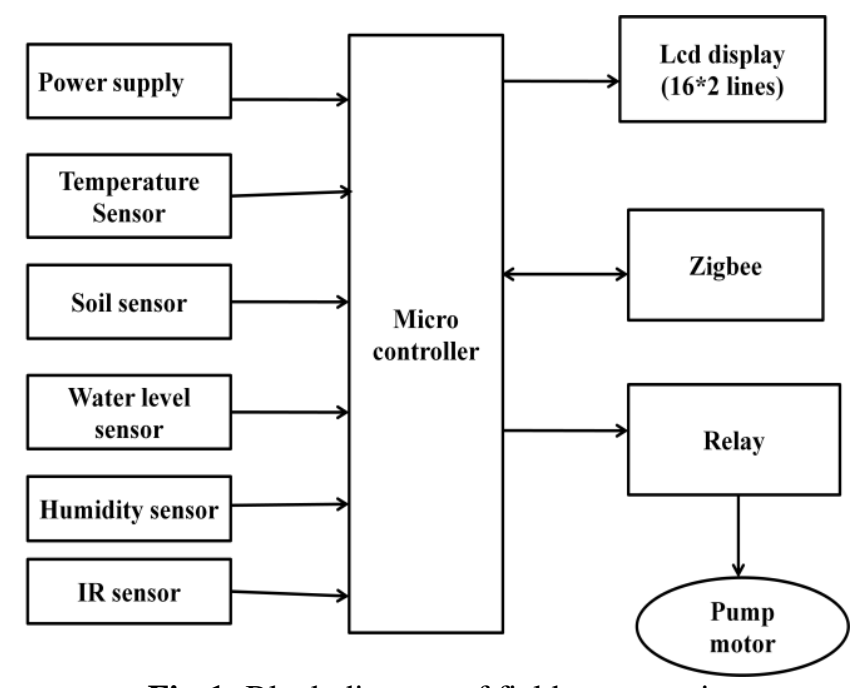

Fig-1: Block diagram of field sensor unit

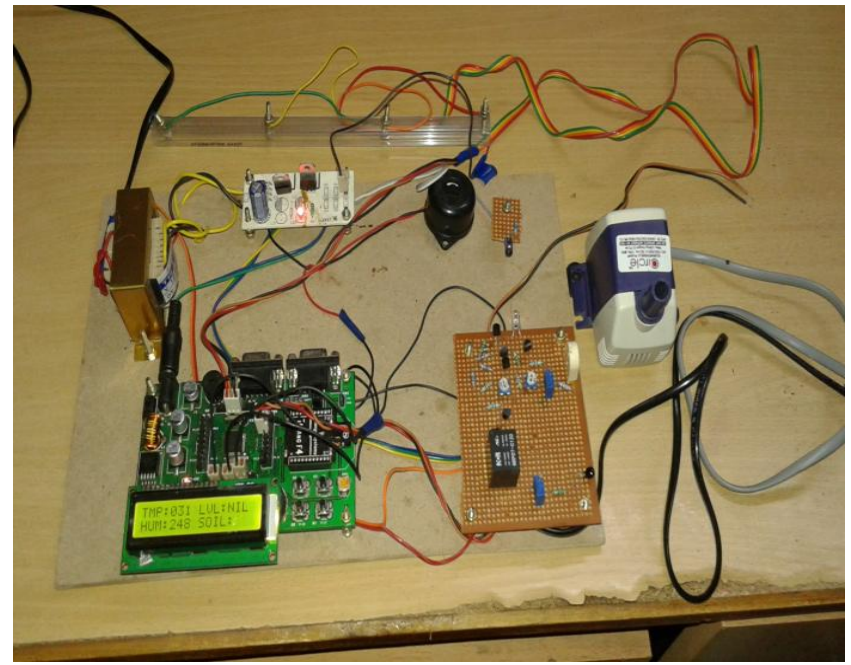

Fig-2: Field sensor unit to monitor status of all the sensors

\subsubsection{LPC2148 Microcontroller}

LPC2148 is the widely used IC from ARM-7 family. It is manufactured by Philips. ARM7 is one of the widely used micro-controller family in embedded system application. The below Fig 3. Shows LPC2148 microcontroller.

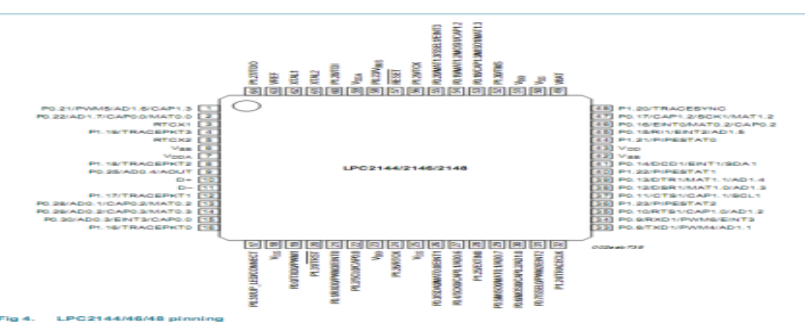

Fig-3:LPC2148 microcontroller

\subsubsection{ZIGBEE Module}

ZIGBEE is a IEEE 802.15.4-based specification for a suite of high-level communication protocols used to create personal area networks with small, low-power digital radios. In our project ZIGBEE is used to send sensor's values from field sensor unit to field information unit.

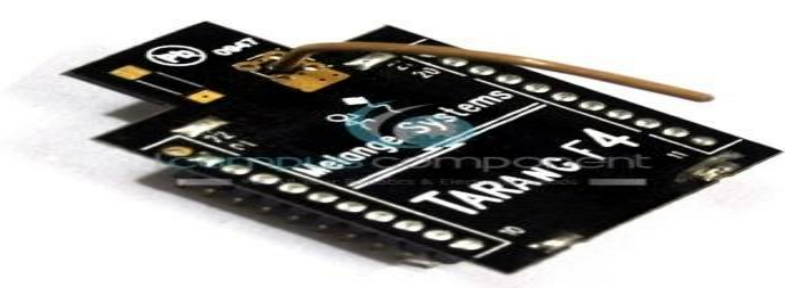

Fig-4: ZIGBEE module

\subsubsection{Liquid Crystal Display}

LCD (Liquid Crystal Display) screen is an electronic display module and find a wide range of applications. A 16x2 LCD display is very basic module and is very commonly used in various devices and circuits. In our project we used two $16 \times 2$ LCDs which are used for displaying status of sensors present in field. 


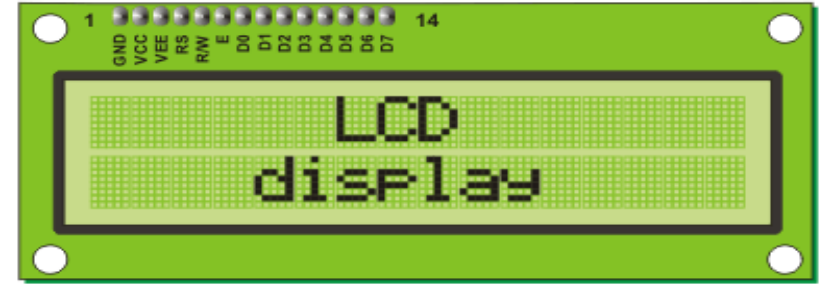

Fig-5: Liquid crystal display

\subsection{Temperature Sensor}

Temperature sensor is used to measure temperature value in the field. In this project we use LM35 temperature sensor. The below fig 6. Shows LM35.

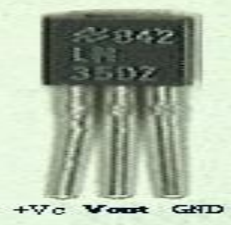

Fig-6: LM35

\subsubsection{Soil Moisture Sensor}

Measuring soil moisture is important for agricultural applications to help farmers manage their irrigation systems more efficiently. In this project we use soil sensor. It is used for checking the soil condition. Whether it is in wet condition or in dry condition. The below fig 7. Shows soil moisture sensor

\section{MOISTUPE SENSOR CIROUIT}

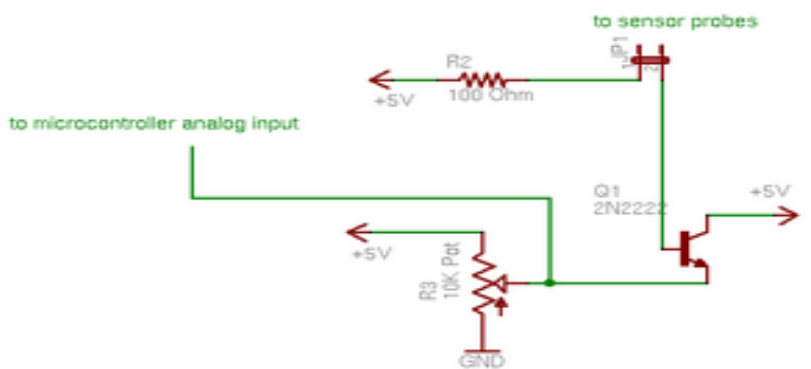

Fig-7: Soil moisture sensor

\subsubsection{Humidity Sensor}

In this project humidity sensor is used. Humidity is the amount of water vapor in the air. The below fig 8 . Shows humidity sensor.

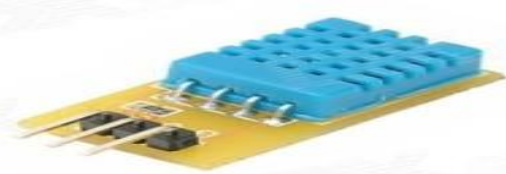

Fig-8: Humidity Sensor

\subsubsection{Water Level Sensors}

Water level sensor is used to measure the water level in tank. Depending up on the level of water in tank motor is ON/OFF. In this paper water level sensor having three levels Low level, Medium level, and High level. If the water level sensor is in below the threshold level i.e. below the low level the motor is in OFF condition. If the water level sensor is in (low or medium or high) levels the motor is in ON condition

\subsubsection{IR sensor}

An infrared sensor is an electronic device that emits and/or detects infrared radiation in order to sense some aspect of its surroundings. Infrared sensors can measure the heat of an object, as well as detect motion. The below fig 9. Shows IR sensor..

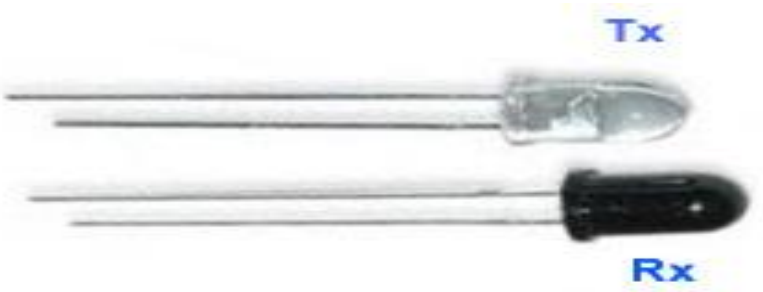

Fig-9: IR sensor

\subsection{Field Information Unit}

This section describes about field information unit. The below Fig 10. Shows the block diagram of a field information unit. The soil moisture sensor, temperature sensor and water level sensor values from each field sensor unit are received, identified, recorded, and analyzed in the field information unit. The field information unit consists of LPC2148 micro- controller, ZIGBEE module, a GPRS module. This processed information i.e., status of all these sensors is send to web page by using GPRS technology and send as SMS by using GSM technology. The below fig 11. Shows the field information unit to observe field conditions remotely.

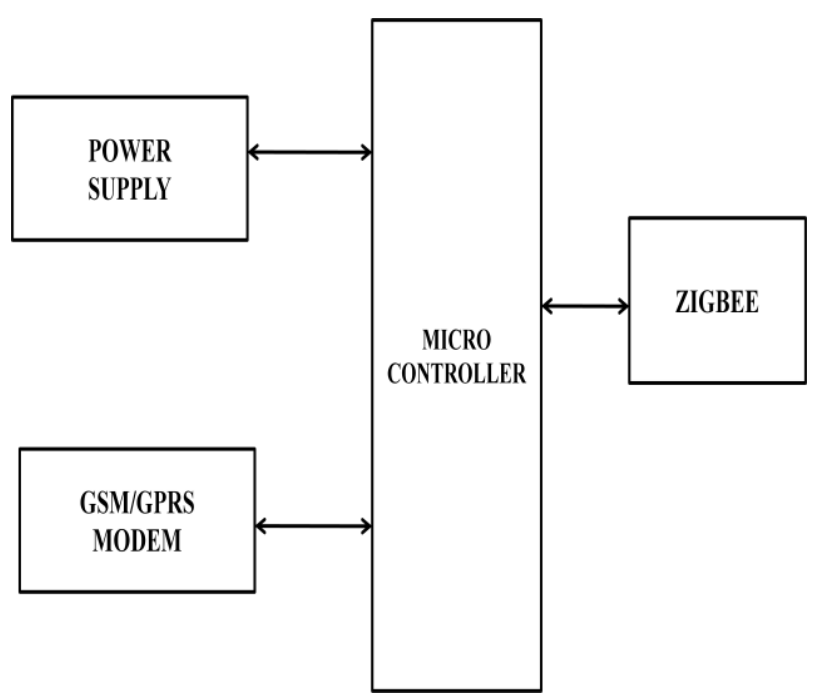

Fig-10: Block diagram of field information unit 


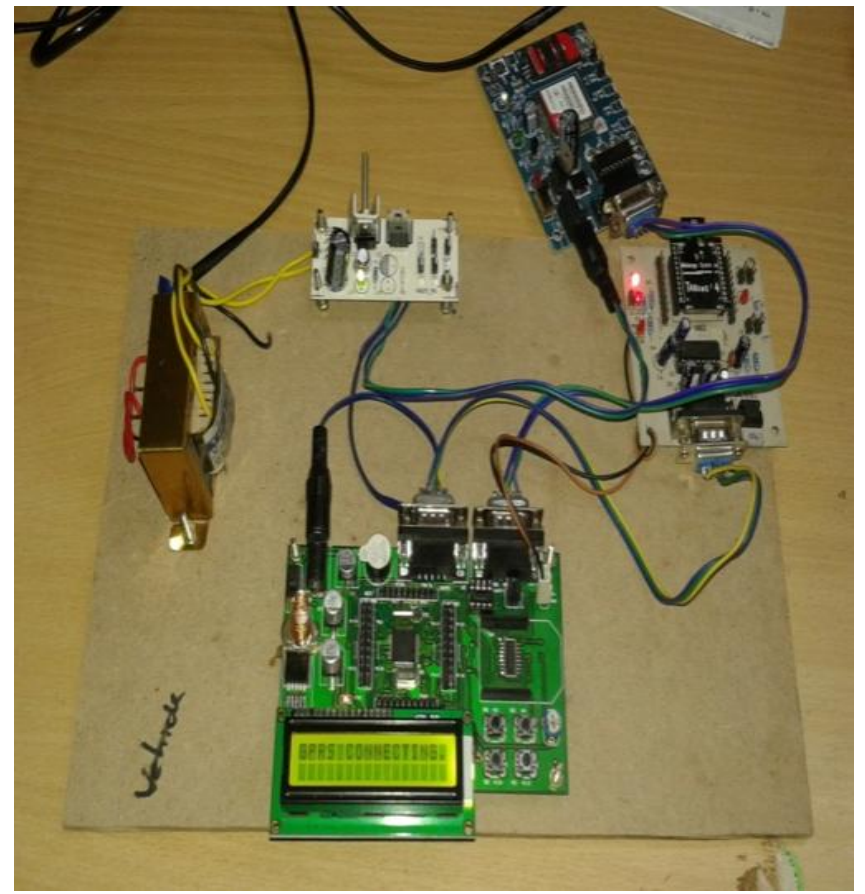

Fig-11: Field information unit to observe field conditions remotely

\subsubsection{GSM Technology}

The GSM standard has given birth to wireless services like GPRS and EDGE. Its main advantage is the implementation of SMS (short message system), which is more popularly known as texting. In our project by using GSM technology we can monitor the status of the sensors. We will get an SMS about status of the sensors by using GSM technology.

\subsubsection{GPRS technology}

General packet radio service (GPRS) is a packet oriented mobile data service on the $2 \mathrm{G}$ and $3 \mathrm{G}$ cellular communication system's global system for mobile communication (GSM)..In this project all sensors information will be send to webpage by using GPRS technology. The below Fig 12. Shows GPRS modem.

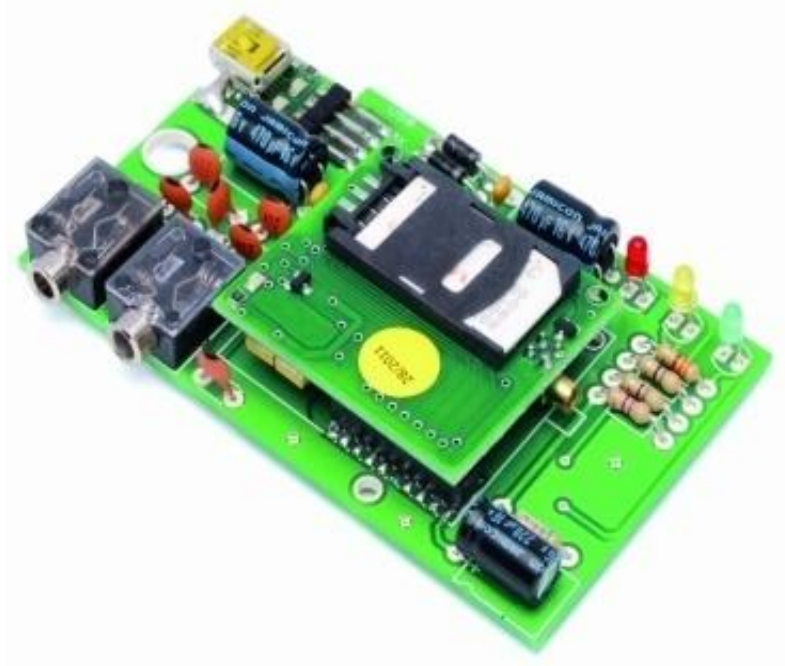

Fig-12: GSM/GPRS modem

\section{WORKING OF THE PROPOSED SYSTEM}

Implementation of agricultural automation system through WEB and GSM technology consists of field sensor unit and field information unit separately. Mainly this project is used for reducing human effort and for optimizes water usage in the field of agriculture automatically. In our system we use the following sensors:

Temperature sensor- it sens the temperature changes.

Soil sensor: - it checks the soil condition. Whether is wet or dry.

Humidity sensor: - it sense the humidity present in the surroundings environment

IR sensor: - it is used for finding the intruder

Water level sensor: - it displays water levels whether water is in low or medium or in high levels

Before switch on the system first we insert SIM in GPRS module that SIM must have the data package activation. For field sensor unit we give $5 \mathrm{~V}$ d.c supply by using power supply circuit. Next switch on the field information unit and GPRS module. For initialization of GPRS module it takes some time and switch on the field sensor unit. In field sensor unit LCD displays as "WELCOME TO THE PROJECT". And it displays temperature, water level, humidity, soil sensor values.

First we check the soil condition in the field. When soil moisture value is less than the threshold value then the motor gets started automatically but at that time water level is also checked. As there is no water in tank then motor remains off. There are different conditions for the automation of the irrigation system depends on the sensor values and these conditions are given in Table: 1.For each and every time these values are displayed by the two LCD s. By using ZIGBEE technology values are transferred from field sensor unit to field information unit. The status of all these values is send to webpage by using GPRS technology and send as a SMS by using GSM technology.

Table-1: Different condition for Agricultural Automation Operation

\begin{tabular}{|c|c|c|c|}
\hline Case & Soil Moisture & Water level & Motor Status \\
\hline 1 & DRY & FULL & ON \\
\hline 2 & WET & FULL & OFF \\
\hline 3 & DRY & MEDIUM & ON \\
\hline 4 & WET & MEDIUM & OFF \\
\hline 5 & DRY & LOW & ON \\
\hline 6 & WET & LOW & OFF \\
\hline 7 & DRY & NULL & OFF \\
\hline 8 & WET & NULL & OFF \\
\hline
\end{tabular}




\section{RESULTS OF THE PROPOSED SYSTEM}

This section describes about final results of a proposed system. Here statuses of all the sensors are monitored by using GSM and GPRS technologies.

\subsection{Status of Field On Webpage By Using GPRS}

\section{Technology}

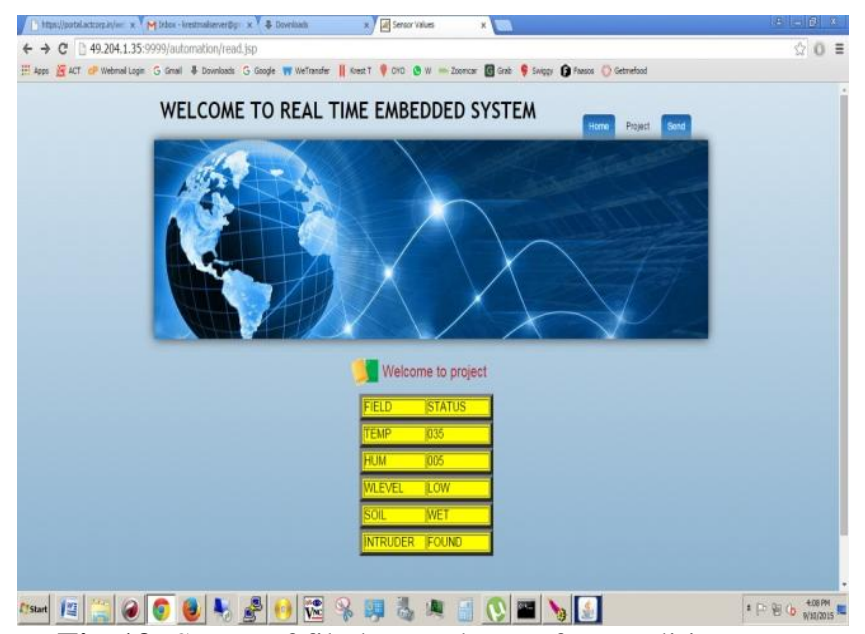

Fig-13: Status of filed on webpage for condition no-6

In the above web page gives the status of the field for condition number 6 in the table1, in that we can monitor the different parameters like the temperature, humidity, water-level, soil, and IR sensor values.

\subsection{Status Of Field As A SMS By Using GSM}

\section{Technology.}

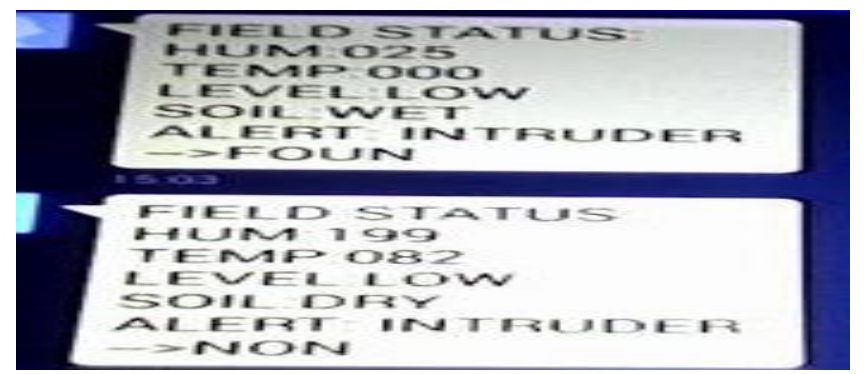

Fig-14: Status of filed as a SMS for condition no- 6 and 7

In the above SMS gives the status of the field for condition numbers 6 and 7 in the table1, in that we can monitor the different parameters like the temperature, humidity, waterlevel, soil, IR sensor values.

\section{CONCLUSION}

Implementation of agricultural automation system using WEB \& GSM technologies proves that it optimizes the water usage because it provides irrigation as per the requirement of the crop and it also reduces the human effort. We can monitor the status of all the sensors (Soil-moisture, Temperature, Water level) by using WEB and GSM technologies

\section{REFERENCES}

[1]. JoaqunGutirrez, Juan Francisco Villa-Medina, Alejandra Nieto- Garibay, And Miguel ngelPortaGndara, "Automated Irrigation System Using A Wireless Sensor Network And GPRS Module", Ieee Transactions On Instrumentation And Measurement, Vol. 63, No. 1, January 2014.

[2]. Vimal P, Priyanka.V, Rajyasree.M, Santhiyadevi P.T, Jagadeeshraja.M, SuthanthiraVanitha.N"A Novel Approach for Automatic Irrigation and Fertigation Using Embedded System," International Journal of VLSI and Embedded Systems-IjvesVol 05, Article 03257; March 2014.

[3]. Sathiyabama P, Lakshmi Priya C, Ramesh Sm, Preethi B, Mohanaarasi M, "Embedded System Design For Irrigating Field With Different Crops Using Soil Moisture Sensor," International Journal Of Innovative Research In Computer And Communication Engineering Vol. 2, Issue 8, August 2014.

[4]. LiaiGao, Meng Zhang, GengChen,"An Intelligent Irrigation System Based On Wireless Sensor Network and Fuzzy Control, "Journal of Networks, Vol. 8, No. 5, May 2013

[5]. Orazio Mirabella, Senior Member, IEEE, and Michele Brischetto,"A Hybrid Wired/Wireless Networking Infrastructure for Greenhouse Management,'IEEETransactionsonInstrumentationand Measurement, Vol. 60, No. 2, February 2011.

[6]. B.Sivakumar, P.Gunasekaran, T.Selvaprabhu, P.Kumaran, D.Anandan, "The Application of Wireless Sensor Network in the Irrigation Area Automatic System", IjctaJan-Feb 2012.

[7]. S.MuhammadUmair, R. Usman,"Automation Of Irrigation System Using Ann Based Controller," International Journal Of Electrical Computer Sciences Ijecs-Ijens Vol: 10 No: 02.May2010.

\section{BIOGRAPHIES}

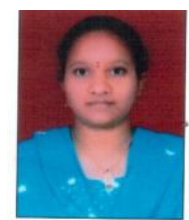

Vidadala srija, pursuing M.Tech (VLSI \& ES) from Sri Mittapalli Institute of Technology For Women, Guntur, A.P, India.

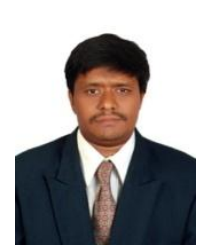

Balamuralikrishna Potti is Professor at Sri Mittapalli Institute of Technology for Women, Guntur, India. He has 15 years of teaching and 4 years of research experience. He received B.E. degree in Electronics and Communication Engineering from Andhra University, India in 2001 and M.Tech. Degree in Instrumentation and control systems from JNTU College of Engineering, Kakinada in 2008.He had guided 8 M.Tech projects and $20 \mathrm{~B}$.Tech projects. He has published several papers in International Journals and conferences. He attended 15 workshops / short-term courses. His areas of interest are Computer Networks, Communications, Image processing, Signal processing and Instrumentation. He is a life member in ISTE and IETE 